\title{
Walrasian economy and some properties of convexly compact sets
}

\author{
Mirela Cristea, Doina Dascălu*, and Laura Nicoleta Nasta
}

\begin{abstract}
G. Žitković defined the notion of a convexly compact set in a topological space and, among other things, used it to give an extension of the Walrasian excess-demand theorem. We continue the study of convexly compactness in LCS spaces and prove a Krein-Milman theorem in this setting.
\end{abstract}

\section{Introduction}

At the end of the $19^{\text {th }}$ century L. Walras [6] defined the state of an economic system at a given moment by using a system of equations involving the demand and the supply, the equilibrium meaning that the supply is the same as the demand. In the 1950's K. J. Arrow and G. Debreu and, independently, L. W. McKenzie developed a model of production, exchange, and consumption in a competitive economy, and, more than that, they proved the existence of an equilibrium point for this model. We will describe below Arrow and Debreu's model constructed in [2]. For a survey of these results and background and subsequent developments, see [5].

The system is based on two principal categories of elements: $n$ production units (typically enterprises) and $m$ consumption units (typically individuals). Also, the economy implies the exchange of a certain number of commodities,

Key Words: convex set, extremal point, Walrasian economy

2010 Mathematics Subject Classification: Primary 46A55, 91B52

Received: 20.02.2016

Accepted: 23.04.2016

*corresponding author 
whose number is $l$ (note that types of labor might also be some of these commodities). As is customary, we will use the letters $y, Y$ for quantities related to production units and the letters $x, X$ for quantities related to consumption units.

We assume then that we have $n$ production units labeled by the set $\{1, \ldots, n\}$. A production unit may produce a certain commodity or consume it; in the first case the commodity is an output of the unit, while in the second it is an input. A production plan for unit $j$ is an vector $y \in \mathbb{R}^{l}$, where the component $y_{j h}, 1 \leq h \leq l$ denotes the output/input of the commodity $h$, where inputs are negative and outputs as positive numbers. We make the notation $\Omega=\left\{x=\left(x_{1}, \ldots, x_{l}\right) \in \mathbb{R}^{l}: x_{i} \geq 0, \forall i\right\}$

It is realistic to assume that not all vectors in $\mathbb{R}^{l}$ are possible production plans for a given unit - there might be constraints of size, capacity, etc. The assumption we make is that each production unit $j$ has an associated set $Y_{j} \subset$ $\mathbb{R}^{l}$ of possible production plans. For $y_{j} \in Y_{j}, y=\left(y_{j 1}, \ldots, y_{j l}\right)$, the component $y_{j h}, 1 \leq h \leq l$ denotes the output/input of the commodity $h$; remember inputs are negative and outputs are positive numbers. Define $Y=\sum_{j=1}^{n} Y_{j}$; then the elements of $Y$ represent the possible output/input values of commodities for the whole production sector. We assume then that $Y_{j}$ satisfy the following three conditions:

I.a For each $j, Y_{j}$ is closed, convex, and $0 \in Y_{j}$.

I.b. $Y \cap \Omega=0$.

I.c. $Y \cap(-Y)=0$.

The different assertions in I.a. are natural. Assumption I.b. says that on the level of the whole production sector there cannot be only outputs without any inputs. Assumption I.c. is a consequence of the fact that at least one of the commodities is labor, which cannot be an output of a production unit.

We pass now to the consumption units, that is, individuals. A vector $x \in \mathbb{R}^{l}$ may be regarded also as a consumption vector for a unit $i$; in this case the component $x_{i h}$ represents the quantity of the commodity $h$ consumed by the individual $i$. Here also it is realistic to assume that, even in the absence of budgetary constraints, not all vectors in $\mathbb{R}^{l}$ may be consumption vectors. Thus for each $i \in\{1, \ldots, m\}$, we denote by $X_{i}$ the set consumption vectors that unit $i$ would choose without any budgetary constraint. Then $X_{i} \subset \mathbb{R}^{l}$ and for a vector $x_{i} \in X_{i}$ and $h \in\{1, \ldots, l\}$, the component $x_{i h}$ represents the quantity of the commodity $h$ consumed by the unit $i$.

The main constraint that has to be satisfied by the sets $X_{i}$ comes from the fact that quantities of consumed non-labor commodities have to be positive (an individual does not produce), while the quantity of labor supplied is bounded by natural factors (capacity, length of time). Since labor supplied is considered negative as a commodity consumed by an individual, there should be a lower 
bound for it. Thus we assume that the set $X_{i}$ satisfies the following condition:

II. $X_{i}$ is closed, convex, and bounded from below, i.e. there exists $\xi_{i} \in \mathbb{R}^{l}$ such that $\xi_{i h} \leq x_{i h}$ for every $x_{i} \in X_{i}$ and every $h \in\{1, \ldots, l\}$.

Another important element is introduced by the preferences of each individual. We assume that each consumption unit $i$ has a preference scale that determines preferences between the consumption vectors. The preference scale of unit $i$ is determined by a function $u_{i}: X_{i} \rightarrow \mathbb{R}$, such that $x$ is preferred to $x^{\prime}$ if and only if $u_{i}(x) \geq u_{i}\left(x^{\prime}\right)$. We assume that the functions $u_{i}$ satisfy the following conditions:

III.a. $u_{i}$ is continuous.

III.b For any $x \in X_{i}$ there exists $x^{\prime} \in X_{i}$ such that $u_{i}\left(x^{\prime}\right)>u_{i}(x)$.

III.c. For $x, x^{\prime} \in X_{i}$, if $u_{i}\left(x^{\prime}\right)>u_{i}(x)$ then $u_{i}\left(t x+(1-t) x^{\prime}\right)>u_{i}(x)$ for $t \in(0,1)$.

Assumptions III.a. and III.c. may be regarded as natural; see, however, [2] for some interesting comments. Assumption III.b. means that there is no "ideally" preferred consumption vector, that some improvement is always possible.

Two more elements characterize the consumption units. First, each consumption unit $i$ is endowed with some initial holdings of the different commodities, defined by a vector $\zeta_{i} \in \mathbb{R}^{l}$. Secondly, we assume that the individual are always shareholders in the production units; then the share of the profit of the $j^{\text {th }}$ production unit that is distributed to the consumption unit $i$ is denoted by $\alpha_{i j}$. We assume that they satisfy the following conditions:

IV.a. There exists $x_{i} \in X_{i}$ such that $x_{i, h}<\zeta_{i, h}$ for all $h \in\{1, \ldots, l\}$.

IV.b. $\sum_{i=1}^{m} \alpha_{i j}=1$ for all $j \in\{1, \ldots, n\}$

The significance of IV.b. is obvious. That of IV.a. is less evident. It says that any individual $i$ could consume something of each commodity, and still have a positive quantity remaining. This turns out in the theory to be a necessary condition for the existence of the searched equilibrium point; it means that this existence implies the fact that each individual should be able to influence each commodity.

Finally, the functioning of the economy implies the existence of a system of prices for each commodity. this is again a vector $p \in \mathbb{R}^{n}$; since prices are positive we have $p_{h} \geq 0$ for all $h$, and we may always assume the normalization condition that the sum of all prices is equal to 1 . So we are considering the set $P=\left\{p=\left(p_{1}, \ldots, p_{l}\right) \in \mathbb{R}^{l}: p_{h} \geq 0, \sum_{h=1}^{l} p_{h}=1\right\}$, and for the price vector $p$ we have $p \in P$. We denote by $\langle\cdot, \cdot\rangle$ the euclidean inner product on $\mathbb{R}^{l}$, so that $\langle x, y\rangle=\sum_{h=1}^{l} x_{h} y_{h}$. 
Definition 1. A competitive equilibrium is an element

$$
\left(x_{1}^{*}, \ldots, x_{m}^{*}, y_{1}^{*}, \ldots, y_{n}^{*}, p^{*}\right) \in \prod_{i=1}^{m} X_{i} \times \prod_{j=1}^{m} Y_{j} \times \mathbb{R}^{l}
$$

that satisfies the following conditions:

1. $y_{j}^{*}$ maximizes $\left\langle p^{*}, y_{j}\right\rangle$ over $y_{j} \in Y_{j}$. This means that each production unit should obtain for its production the maximum total price possible.

2. $x_{i}^{*}$ maximizes $u_{i}\left(x_{i}\right)$ when $x_{i} \in\left\{x \in X_{i}:\left\langle p^{*}, x\right\rangle \leq\left\langle p^{*}, \zeta_{i}\right\rangle+\sum_{j=1}^{n}\left\langle p^{*}, y_{j}^{*}\right\rangle\right\}$.

This represents the maximization of the preference functions for each consumption unit. The supplementary condition, besides $x \in X_{i}$, stipulates that the consumption is limited by the initial holdings plus the share of the production units that are attributed to the given consumption unit.

3. $p^{*} \in P$.

4. If $z^{*}=\sum_{i=1}^{m} x_{i}^{*}-\sum_{j=1}^{n} y_{j}^{*}-\sum_{i=1}^{m} \zeta_{i}$ then $z_{h}^{*} \leq 0$ for every $h \in\{1, \ldots, l\}$ and $\left\langle p^{*}, z^{*}\right\rangle=0$. A detailed analysis of condition shows that it allows the possibility that if a commodity has zero price, then supply may exceed demand. This is not possible for a nonzero price, since the excess would drive the price down; but the fact that prices have to be nonnegative makes necessary this slight complication.

Then the main Theorem 1 in [2] states the existence of an equilibrium point:

Theorem 1. If an economic system satisfies conditions $I$ - IV, then there exists an equilibrium point.

The setting above was finite dimensional, hence locally compact. The equilibrium theory for an economic system has been also considered in the more general setting of infinite-dimensional spaces. For a survey, see [4]. In order to obtain competitive equilibrium in infinite-dimensional spaces, G. Žitković introduced in [7] the notion of a convexly compact set (see Definition 2) and proved a Walrasian excess-demand theorem in topological spaces. In this context he proved the following theorem (Theorem 4.11 in [7]):

Theorem 2. Suppose that $X$ is a topological vector space and let $C$ be a convexly compact subset of $X$. We assume that $D \subset C$ is convex and closed and the function $F: C \times D \rightarrow \mathbb{R}$ satisfy the following conditions:

1. $\{x \in C: F(x, y) \leq 0\}$ is closed and convex for each $y \in D$.

2. $F_{x}: D \rightarrow \mathbb{R}, F_{x}(y)=F(x, y)$ is concave, and for each $x \in C$.

3. $F(y, y) \leq 0$ for each $y \in D$.

Then there exists $x_{0} \in C$ such that $F\left(x_{0}, y\right) \leq 0$, for every $y \in D$. 
Convexly compactness has thus been shown to be an important notion for the economy of infinite-dimensional systems. The purpose of this paper is to understand how far this notion is from compactness. The main result of this paper, see Theorem 3 below, is that the well-known Krein-Milman theorem holds for convexly compact sets. Remember that the original Krein-Milman theorem says that in a LCS (locally convex space) every compact and convex set is the closure of the convex hull of its extremal points. A version of this theorem for cones (the cones, obviously, are not compact) is due to Choquet. Applications to economic theory can be found, for example, in [3].

\section{Results}

Definition 2. Suppose that $X$ is a topological vector space and $C$ is convex subset of $X . C$ is called convexly compact if the following is true:

If a family $\left\{F_{i}\right\}_{i \in I}$ of convex subsets of $C$ which are relatively closed in $C$ is such that for any finite subset $J \subset I$ we have $\bigcap_{j \in J} F_{j} \neq \emptyset$, then $\bigcap_{i \in I} F_{I} \neq \emptyset$.

Notations. If $X$ is a vector space and $A$ is a subset of $X$ space we denote by $\operatorname{co}(A)$ the convex hull of $A$ and if $C$ is a convex subset of $X$ we denote by $\operatorname{ext}(C)$ the set of extremal points of $C$. Remember that $a \in C$ is called an extremal point for $C$ if whenever $x, y \in C$ and $\alpha \in[0,1]$ are such that $a=\alpha x+(1-\alpha) y$ we must have that $a=x$ or $a=y$. A subset $S \subset C$ is called extremal if whenever $x, y \in C, \alpha \in(0,1)$ are such that $\alpha x+(1-\alpha) y \in S$ then we must have $x \in S$ and $y \in S$.

If $X$ is a topological space and $A$ is a subset of $X$ we denote by $\bar{A}$ the closure of $A$.

Lemma 1. Suppose that $X$ is a LCS. If $C \subset X$ is a compactly convex set, then $C$ is closed.

Proof. Suppose that $C$ is not closed and let $x \in \bar{C} \backslash C$. Let $\left\{V_{i}\right\}$ a fundamental system of neighborhoods for $x$ which are closed and convex. Then $F_{i}:=V_{i} \cap C$ are convex and relatively closed in $C, \bigcap_{i \in I} F_{I}=\emptyset$, but $\bigcap_{j \in J} F_{j} \neq \emptyset$ for any finite subset $J \subset I$. The contradiction obtained proves the lemma.

Lemma 2. Suppose that $X$ is a LCS, $C \subset X$ is a compactly convex subset of $X$ and $L: X \rightarrow \mathbb{R}$ is a linear continuous functional. Then $L_{\mid C}$ is bounded.

Proof. For $n \in \mathbb{N}$ let $F_{n}=\{x \in C: L(X) \geq n\} . F_{n}$ are closed and convex, $F_{n} \subset F_{m}$ for $n>m$ and $\cap_{n \geq 1} F_{n}=\emptyset$. It follows that there exists $n_{0}$ such that $F_{n_{0}}=\emptyset$ and therefore $L_{\mid C}<n_{0}$. Similarly $L_{\mid C}$ is bounded from below. 
Lemma 3. Suppose that $X$ is a LCS, $C \subset X$ is a compactly convex subset of $X$ and $L: X \rightarrow \mathbb{R}$ is linear and continuous function. Then $L$ attains its maximum on $C$.

Proof. Let $\alpha=\sup _{C} L(x)$. Then, by Lemma 2, $\alpha$ is finite. Let $F_{n}=\{x \in C$ : $\left.L(X) \geq \alpha-\frac{1}{n}\right\}$. By the definition of $\alpha, F_{n} \neq \emptyset$ for all $n \geq 1$. Also $F_{n}$ are closed and convex and $F_{n} \subset F_{m}$ for $n>m$. It follows that for any $A \subset \mathbb{N}$, $A$ finite, the intersection $\cap_{n \in A} F_{n}$ is not empty. As $C$ is compact convex we deduce that $\cap_{n \in \mathbb{N}} F_{n} \neq \emptyset$. Therefore there exists $x \in C$ with $L(x)=\alpha$.

The next theorem is the analogue, for convexly compact sets, of the classical Krein-Milman theorem.

Theorem 3. Suppose that $X$ is a $L C S, C \subset X$ is a compactly convex subset of $X$. Then $C=\overline{\operatorname{co}(\operatorname{ext}(C))}$.

Proof. First we prove that every compactly convex set $C$ has an extremal point. Let $(\mathcal{K}, \leq)$ be the set of non-empty extremal closed convex subsets of $C$ with the order given by $K_{1} \leq K_{2}$ if and only if $K_{2} \subset K_{1}$. We want to show that $(\mathcal{K}, \leq)$ has a maximal element and we apply Zorn's Lemma. Let $\left\{K_{i}\right\}_{i \in I}$ be a chain. By the definition of convex compactness $\cap_{i \in I} K_{i} \neq \emptyset$. Obviously it is extremal and convex. Let $K_{0}$ be a maximal element. We claim that $K_{0}$ consists of just one point. Suppose not and let $x_{1} \neq x_{2} \in K_{0}$. Let $L: X \rightarrow \mathbb{R}$ be linear and continuous such that $L\left(x_{1}\right)<L\left(x_{2}\right)$ (which exists by the Hahn-Banach theorem). Note that $K_{0}$ is closed and convex compact. Let $\alpha=\max _{K_{0}} L(x)$ which exists by Lemma 3 and $K_{0}^{\prime}=K_{0} \cap\{x: L(x)=\alpha\}$. It follows that $K_{0}^{\prime}$ is convex and extremal for $K_{0}$ and hence for $C, K_{0}^{\prime} \subset K_{0}$ and as $x_{1} \notin K_{0}^{\prime}$ we have also that $K_{0}^{\prime} \neq K_{0}$. This contradicts the maximality of $K_{0}$.

We prove now that $C=\overline{\operatorname{co}(\operatorname{ext}(C))}$. Suppose now that this is not the case and let $x_{0} \in C \backslash \overline{\operatorname{co}(\operatorname{ext}(C))}$. Note that $\overline{\operatorname{co}(\operatorname{ext}(C))}$ is convex compact. Let $L: X \rightarrow \mathbb{R}$ be linear and continuous such that $L\left(x_{0}\right)>\max \{L(x): x \in$ $\overline{\operatorname{co}(\operatorname{ext}(C))}\}$. Let $C^{\prime}=\max \{L(x): x \in C\}$. Then $C^{\prime}$ is extremal for $C$, it is closed and convex compact, and, at the same time, $C^{\prime} \cap \overline{\operatorname{co}(\operatorname{ext}(C))}=\emptyset$. By the first part of the proof $C^{\prime}$ has and extremal point $x_{1}$. As $C^{\prime}$ is extremal for $C$, we have that $x_{1}$ is an extremal point for $C$ as well which is impossible since $x_{1} \notin \overline{\operatorname{co}(\operatorname{ext}(C))}$

The following examples are relevant for the relation between compactness and convexly compactness in locally convex spaces.

\section{Examples.}

1. Let $C \subset \mathbb{R}^{n}$ be a convex subset. Then $C$ is convexly compact if and only if it is compact. This follows from Lemma 1 and Lemma 2. 
2. If $X$ is a reflexive Banach space then the closed unit ball is convexly compact. This follows from Alaoglu Theorem and the fact that a convex set is closed in the norm topology if and only if is closed in the weak topology.

3. The closed unit ball in $l_{1}$ is not convexly compact. This follows from Theorem 3 and from the fact that the closed unit ball in $l_{1}$ has no extremal point.

Remark. A non-empty subset $S$ of convex set $C$ in a vector space $X$ is called semi-extremal if $C \backslash S$ is convex. This is the same as saying that if $x, y \in C$, $\alpha \in[0,1]$, and $\alpha x+(1-\alpha) y \in S$ then we must have $x \in S$ or $y \in S$. Andersen and Poulsen [1] proved that if $X$ is a LCS, $C$ is a compact convex subset and $S \subset C$ is closed and semi-extremal then $S \cap \operatorname{ext}(C) \neq \emptyset$. The same proof works in the convexly compact setting. Hence one obtains the following statement: if $X$ is a LCS, $C$ is a convexly compact subset and $S \subset C$ is closed and semi-extremal then $S \cap \operatorname{ext}(C) \neq \emptyset$.

\section{References}

[1] T. B. Andersen; E. T. Poulsen: On semi-extremal subsets of convex sets. Math. Scand. 23 (1968) 167-168.

[2] K. J. Arrow; G. Debreu: Existence of an equilibrium for a competitive economy. Econometrica 22 (1954), 265-290.

[3] Border, K. C. Functional analytic tools for expected utility theory. Positive operators, Riesz spaces, and economics (Pasadena, CA, 1990), 69-88, Springer, Berlin, 1991.

[4] A. Mas-Colell, W. R. Zame: Equilibrium theory in infinite-dimensional spaces. Handbook of mathematical economics, Vol. IV, 1835-1898, Handbooks in Economics, 1, North-Holland, Amsterdam, 1991.

[5] L. W. McKenzie: The classical theorem on existence of competitive equilibrium. Econometrica 49 (1981), 819-841.

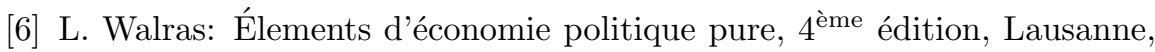
Paris, 1900.

[7] G. Žitković: Convex compactness and its applications. Math. Financ. Econ. 3 (2010), 1-12. 
WALRASIAN ECONOMY AND SOME PROPERTIES OF CONVEXLY COMPACT SETS

Mirela Cristea

University of Craiova

Craiova, Romania

E-mail address: Mirelas.Cristea@gmail.com

Doina Dascălu

Romanian Court of Accounts

Bucharest, Romania

E-mail address: Doina.Dascalu@rcc.ro

Laura Nicoleta Nasta

Bucharest University of Economic Studies

Bucharest, Romania 